\title{
Predicting the Reasons of Customer Complaints: A First Step Toward Anticipating Quality Issues of In Vitro Diagnostics Assays with Machine Learning
}

Stephane Aris-Brosou ${ }^{1}, \mathrm{PhD}$; James $\mathrm{Kim}^{2}, \mathrm{MBA} ; \mathrm{Li} \mathrm{Li}^{2}, \mathrm{MD}, \mathrm{PhD}$; Hui Liu ${ }^{2}, \mathrm{PhD}$

${ }_{1}^{1}$ Department of Biology, University Of Ottawa, Ottawa, ON, Canada

${ }^{2}$ Ortho Clinical Diagnostics, Raritan, NJ, United States

Corresponding Author:

Stephane Aris-Brosou, $\mathrm{PhD}$

Department of Biology

University Of Ottawa

30 Marie Curie Pvt.

Ottawa, ON, K1N 6N5

Canada

Phone: 16135625800

Fax: 16135625486

Email: sarisbro@uottawa.ca

\section{Abstract}

Background: Vendors in the health care industry produce diagnostic systems that, through a secured connection, allow them to monitor performance almost in real time. However, challenges exist in analyzing and interpreting large volumes of noisy quality control (QC) data. As a result, some QC shifts may not be detected early enough by the vendor, but lead a customer to complain.

Objective: The aim of this study was to hypothesize that a more proactive response could be designed by utilizing the collected QC data more efficiently. Our aim is therefore to help prevent customer complaints by predicting them based on the QC data collected by in vitro diagnostic systems.

Methods: QC data from five select in vitro diagnostic assays were combined with the corresponding database of customer complaints over a period of 90 days. A subset of these data over the last 45 days was also analyzed to assess how the length of the training period affects predictions. We defined a set of features used to train two classifiers, one based on decision trees and the other based on adaptive boosting, and assessed model performance by cross-validation.

Results: The cross-validations showed classification error rates close to zero for some assays with adaptive boosting when predicting the potential cause of customer complaints. Performance was improved by shortening the training period when the volume of complaints increased. Denoising filters that reduced the number of categories to predict further improved performance, as their application simplified the prediction problem.

Conclusions: This novel approach to predicting customer complaints based on QC data may allow the diagnostic industry, the expected end user of our approach, to proactively identify potential product quality issues and fix these before receiving customer complaints. This represents a new step in the direction of using big data toward product quality improvement.

(JMIR Med Inform 2018;6(2):e34) doi: 10.2196/medinform.9960

\section{KEYWORDS}

post market surveillance; QC chemistry results; complaint data; CART; adaptive boosting

\section{Introduction}

Connected and so-called smart meters and other tools have transformed virtually every industry by enabling new functions and capabilities such as continuous monitoring, control, optimization, and autonomy [1]. This is particularly true in the health care industry, which deployed analytical systems ranging from electronic health records (EHRs) to clinical decision support systems [2]. Connected systems also include in vitro diagnostic (IVD) analyzers, which work with different assays 
that measure a number of markers in patients' blood samples such as sodium or potassium, as well as other biomarkers such as troponin-which altogether are called "assays." Being connected, their manufacturers can monitor the analyzers' output in real time through encrypted, two-way interactive connections. As such, manufacturers can potentially quickly detect issues and act promptly to resolve the problem.

However, the sheer amount of data generated by these connected systems is such that big data analytics are required [3]. For this, a number of platforms have been developed, going from statistical tools such as R [4], to dedicated business intelligence and data mining tools. These platforms can then generate queries, reports, and perform online analytics processing, as well as data mining [2]. These aggregated data can then be used to perform one of three kinds of analytics: (1) descriptive analytics that permit the visualization of the data; (2) predictive analytics that try and predict the future of a system from its past behavior; and (3) prescriptive analytics that make recommendations about the best way to resolve a particular issue [5]. However, different health analytics contexts may require different approaches, as in the case of quality control (QC) data logged by analyzers.

As QC data are routinely used to monitor the performance of IVD and identify signals that may indicate a performance change, a number of approaches have been developed. These range from panels of experts that submit monthly reports [6], to automated systems that resort to summary statistics computed over temporal windows [7-11]. Although simple linear models can be used to monitor these complex systems [12], machine-learning algorithms have already proved capable of generating highly accurate predictions $[13,14]$. However, past approaches mostly have explored simple tools such as decision trees and other standard classifiers [15] and have not (1) Explored more sophisticated algorithms such as adaptive boosting [16] and (2) In the context of noisy and moderately large dataset-that are, hence, not always amenable to deep learning as recently deployed in the context of EHRs [17]. One aspect that has rarely been integrated into the analysis of QC data is its relation with customer data: when a shift in performance of a test assay is identified, what is its impact on the user (customer)? Will this trigger a complaint about QC? If the complaint is specific, such as "QC high" or "accuracy low," can we learn something about the quality of the data from the combination of those specific complaints?

The objective here is therefore to integrate these two kinds of data, QC data and customer complaints, to be able to predict specific QC issues, while accounting for intrinsic issues pertaining to customer data. Indeed, customer complaint databases have at least three inherent limitations that need to be considered when designing a prediction tool. First, complaint databases may contain inaccurate, incomplete, untimely, or unverified information [18]. Second, incidence may be under $[19,20]$ or overreported [21]. For instance, certain advertising or regulatory actions may result in increased reporting [22], which could ultimately result in an overwhelming important signal with noise. Third, despite the best efforts of complaint handling professionals, errors while curating complaints (eg, misclassification of complaints) occur [23]. However, it is possible that by focusing solely on errors directly related to QC, or even by binning particular errors into larger categories (eg, "QC high" and "QC shift high" in the same category), it might alleviate some of these reporting issues.

Here, based on a particular connected IVD analyzer, we show that integrating QC data with a database of customer complaints can be used to predict which type of issues customers complain about. We hypothesized that connected systems can be utilized more efficiently by utilizing the collected QC data more efficiently and more specifically by resorting to machine-learning algorithms. We show that it is possible to identify product issues more proactively, which makes it possible to act on these before they trigger a customer complaint. We further show that some filtering of the complaint data (denoising) improves the accuracy of issues prediction. This work represents a first step toward meeting the recent plan from the US Food and Drug Administration (FDA) to leverage on big data to improve device performance and health care [24].

\section{Methods}

\section{Data Collection}

\section{e-Connectivity Data}

Data were collected using the e-Connectivity application's chemistry results, manufactured by Ortho Clinical Diagnostics (Raritan, New Jersey). This feature allows the manufacturer to pull information remotely from equipment installed at customer sites, which are themselves distributed throughout the world. The data retrieved in this study were generated by Ortho Clinical Diagnostics' VITROS analyzers of the 5,1 FS series, the 5600, 4600, 3600, or ECi/ECiQ Systems, that all log the same kind of information through e-Connectivity. Only QC data were extracted to avoid complications linked to patients' data (identifiability, variability, etc).

The e-Connectivity data contain information relative to the assay, serial numbers reflecting its origin, the measured concentrations, as well as some information relative to the analyzer itself (see Table 1 and Multimedia Appendix 1 for a full description of the e-Connectivity variables). We focused on five assays, here recoded as "assay A" to "assay E." The data pulled ranged from March 16, 2016 00:00:20 EST to June 14, 2016 23:38:51 EST, a total of 90.98 days, and contained 824,885 QC $\operatorname{logs}$ across the five assays. To assess the effect of the training period, we constructed a second set of data limited to the last 45 days of this 90-day set.

\section{Customer Data}

The corresponding customer complaint data were obtained by querying the product complaint database of the same manufacturer for the same time window as the QC data. Customer data contained information with respect to the assay for which an issue is reported, the call area (error code), and other information related to the assay (see Table 1 and Multimedia Appendix 2 for a full description of the customer variables; Multimedia Appendix 3 list the call areas reported over the five assays employed here). These data contained a total of 7999 logs. Across the five assays tested here, a total of 
99 call areas were found. The goal here is to predict these call areas from the QC data.

\section{Records Matching}

The only fields that are shared between QC and customer data are assay name, $\mathrm{J}$ numbers, and lot numbers (Table 1). As each analyzer has a unique $\mathrm{J}$ number, we used this shared information to match QC samples with customer data. Although this approach works in most cases, there are instances when the same customer processes multiple samples, potentially from multiple analyzers, but logs only one call. Thus, the data that will be used to train the predictive algorithms are, in essence, noisy.

\section{Predictive Classifiers}

\section{Feature Definitions}

To find predictors of customer complaints based on QC data, we need to define operational variables, which are called features. These features were defined by inspecting a typical $\log$ of the system (Figure 1). From this, two types of features were defined, based on (1) concentration readings and (2) maintenance events (eg, change of calibration). 
Table 1. List of the fields logged by e-Connectivity (that includes quality control, QC data) and by the customer complaint system. Corresponding abbreviations are shown.

\begin{tabular}{|c|c|}
\hline Data and Abbreviation & Short description \\
\hline \multicolumn{2}{|l|}{ e-Connectivity } \\
\hline Assay & Abbreviation of assay name (recoded here) \\
\hline J Number & Unique identifier assigned to each analyzer placed \\
\hline F Concentration & Concentration of solute (assay); $\mathrm{QC}^{\mathrm{a}}$ result \\
\hline Units & Unit of measured concentration $(\mathrm{mmol} / \mathrm{L})$ \\
\hline F Concentration (SI) & Concentration of solute (assay); QC result \\
\hline Units SI & Unit of measured concentration (SI) \\
\hline Reagent Lot Number & Reagent lot number \\
\hline S Gen & Manufacturing generation number \\
\hline S Lot & Manufacturing lot number \\
\hline ERF Lot & Electrolyte reference fluid lot \\
\hline IWF Lot & Immuno-wash fluid lot number \\
\hline Control Lot Number & Performance verifier lot number \\
\hline Cal Curve ID & Calibration curve ID \\
\hline Result ID & Unique identifier (encrypted) of QC result \\
\hline Sample Name & Unique identifier (encrypted) of sample name \\
\hline Time Metering & Time stamp of concentration log through e-Connectivity \\
\hline Total Dilution & Dilution factor \\
\hline Operator Dilution & Operator requested dilution \\
\hline Body Fluid & Fluid type (serum, plasma, or urine) \\
\hline \multicolumn{2}{|l|}{ Customer } \\
\hline Create Audit Date & Time stamp of when complaint was placed \\
\hline Call Subject & Same as assay in e-Connectivity \\
\hline Call Area & Classification of concern or problem of the product or the analyzer-generated condition \\
\hline Resolution & Term describing how the complaint was resolved \\
\hline Complaint Number & Unique identifier of each complaint \\
\hline Customer Number & Unique identifier of each customer \\
\hline J Number & Analyzer serial number \\
\hline Lot number & Reagent lot number \\
\hline Region & Geographic region where complaint was placed \\
\hline Call Status & Current call status of complaint (closed or open) \\
\hline Problem description & Free-text field describing the complaint \\
\hline
\end{tabular}

${ }^{\mathrm{a} Q C}$ : quality control. 
Figure 1. Feature definitions based on a typical sample logged in e-Connectivity. Assay concentrations (here for assay A) are plotted as a function of time. Horizontal blue lines show the modes of the density of sample means (our estimated verifiers). Vertical gray lines show timing of maintenance activities (change of calibration curves, etc). The orange vertical line shows when the customer placed a call—for "accuracy high" (ACCH; indicates the measured concentration is suspected of being higher than the actual value) in this example. The concentration reading just before this call ("\#1") and 10 e-Connectivity logs before it ("\#10") are indicated in red. Our machine-learning (ML) algorithms (in red) aim at learning the signatures (in purple) of call areas (orange) from a training set, to be able to identify those call areas, before a customer complains.

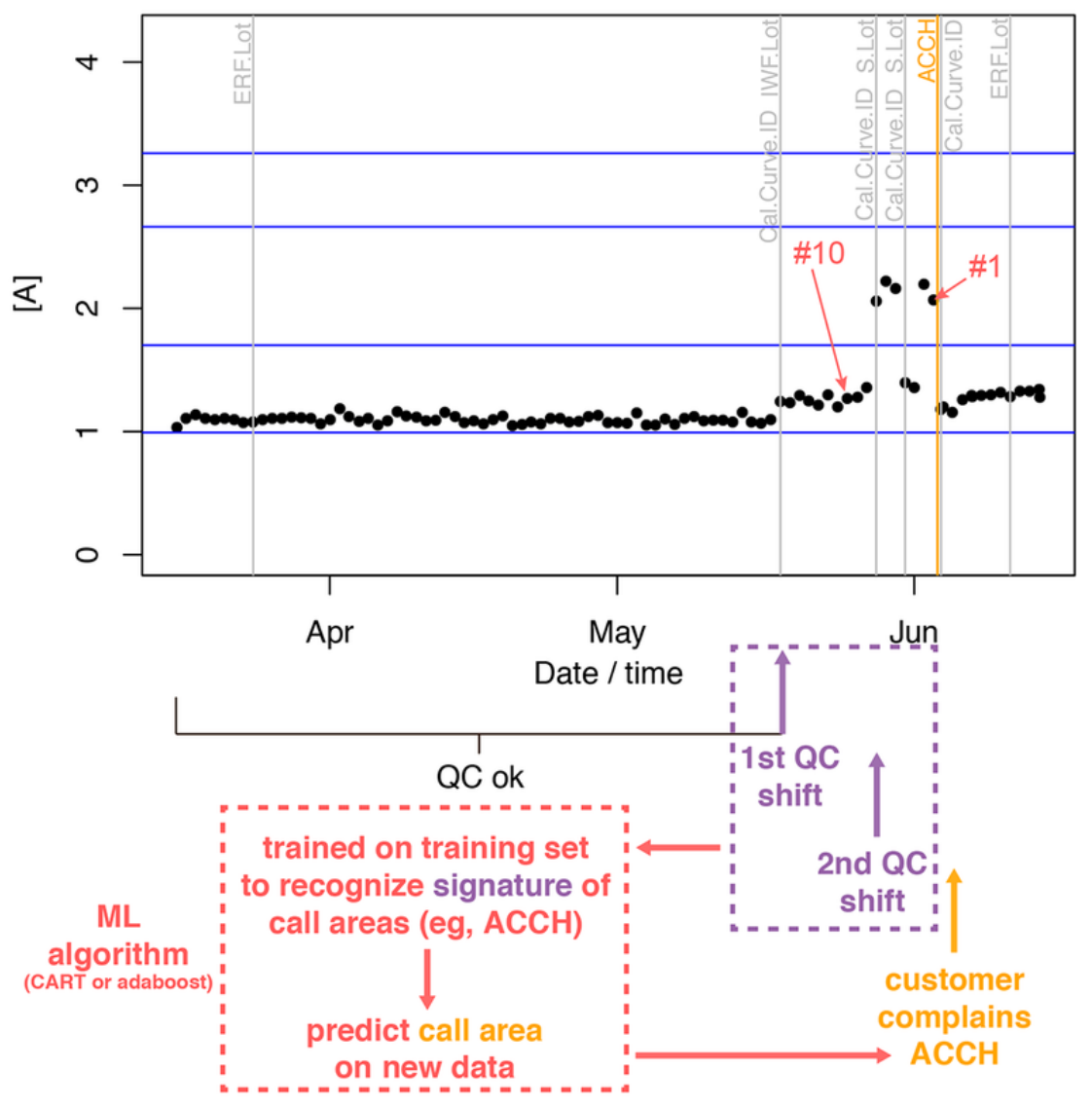

Concentration readings departing from expected values can be thought of as the prime trigger of customer complaints. Obviously, their absolute value with no other context has no predictive value (as long as it is not outside of the biological range) for QC data, and therefore, we should focus on departure from verifiers, which are known concentration readings produced during manufacturing. However, these verifiers are not logged by e-Connectivity and are only available as PDF files, which cannot be easily parsed. Customers may also choose to use QC material that is manufactured by a third-party, which further complicates the retrieval of verifier information. As a workaround, we calculated mean concentrations by samples, estimated the density kernel of these sample means, and determined the location of all the modes (Multimedia Appendix 4). We assumed that each mode corresponds to a verifier: the closest mode to each QC reading logged in e-Connectivity was assumed to be the verifier concentration. We then used the relative differences between concentration readings and estimated verifier. On the basis of this, we defined four features according to concentration readings just before customer call (log “\#1" in Figure 1) and of the average logs two, five, and ten time points before the call (orange vertical bar in Figure 1). Because variability of QC logs may also signal issues, we defined three more features by SDs of the two, five, and ten concentration logs before customer call.
Customers may notice suboptimal performance of a machine and decide to try and resolve the issue on their own and place a call for assistance only if they cannot resolve the issue. We therefore defined features based on different maintenance events logged by the system (six in total): change of S Gen, S Lot, ERF Lot, IWF Lot, Control Lot Number, and Cal Curve ID. We considered both the timing of the last event before the call and the number of such events before the call. This led us to define 12 additional features based on maintenance events, for a grand total of 19 features (Table 2). A twentieth feature was defined as the time it takes for a customer to call since the last e-Connectivity reading (at “\#1” in Figure 1).

Because the use of only "positive samples" (samples that led to a customer call) to train our algorithms would bias any prediction toward overpredicting calls, we also defined features for "negative samples." These are QC samples that did not generate any customer complaints. If $n$ calls were logged for a given assay (among the 7999 logs in total), we drew $n$ such negative samples. We calculated the features as above by drawing a cutoff time at random (from a uniform distribution limited by start and end time of QC logs for a given sample) that plays the role of a customer call in the positive samples. In this case, the call area (error code) is "OK"-giving then a total of 100 call areas that we want to predict. 


\section{Machine-Learning Algorithms}

These 20 features were used as predictors during the training of machine-learning algorithms, whose goal was to classify (predict) the qualitative nature of problem represented by each call area. Two such algorithms were used here: a simple one, based on decision trees [25], and a more sophisticated one that recently proved very successful in one of our applications [26].

Decision trees represent one of the simplest type of classifier, with Classification and Regression Trees (CART) being one of the most basic algorithms. We employed the algorithm implemented in the tree library [27] version 1.0-37 in R version 3.2.3 [4]. Unlike CART, adaptive boosting relies on an iterated process that proposes boundaries in the space of predictors, each giving rise to a weak classifier; the final classifier then combines these different weak classifiers, emphasizing misclassifications, to create a final strong classifier [16]. The adabag library version 4.1 [28] was used. To avoid overfitting with both algorithms, each dataset (the 90- and the 45-day sets) was split into two subsets, where two-thirds of the data were used as a training set and the remaining one-third used to test performances (compute misclassification or error rate from the confusion table). Because of the many ways to split the data in a 2:1 ratio, we repeated this cross-validation exercise for 2500 random such 2:1 splits of the data, for both classifiers. Such a cross-validation experiment can also be seen as a means to prevent overfitting the data with a complex model.

\section{Data Denoising}

Over the 99 call areas employed so far, some are not directly related to QC, and those related to QC might share some characteristics. Both issues can create some noise, which can easily be filtered out of the data. We therefore created two filters, one that removes all non-QC related call areas (essentially, all error codes starting with a "Z" in Multimedia Appendix 3, as they are related to a misconfiguration of the analyzer) and one that bins some call areas. The first filter reduced the complaint data from 7999 to $572 \operatorname{logs}$ and from 99 to 21 call areas by eliminating error codes unrelated to QC. The second filter, binning all QC high (QC high, QC Drift High, QC Shift High) together and all QC low (QC low, QC Drift Low, QC Shift Low) together, further reduced the number of call areas from 21 to 17. Applied both to the 90- and the 45-day data, these filtering steps led to four additional datasets. Our expectation was that these denoising steps would improve the performance of our classifiers, as reducing the number of categories from 99 to 17 simplifies the classification problem. The $\mathrm{R}$ code developed in this study is available from GitHub (sarisbro account); the QC data we used are proprietary, contain no patients records, but the variables used are listed in Table 1.

Table 2. List of the features used in the predictive modeling. Note that a "cutoff" represents the time when a customer calls in the case of "positive samples" (when there is an actual complaint), or the time drawn at random in the case of "negative samples" (see Methods).

\begin{tabular}{|c|c|}
\hline Feature name & Definition \\
\hline MostRecentConcentration & Assay concentration reading just before cutoff \\
\hline TwoMostRecentConcentrationMean & Mean concentration for the two readings before cutoff \\
\hline FiveMostRecentConcentrationMean & Mean concentration for the five readings before cutoff \\
\hline TenMostRecentConcentrationMean & Mean concentration for the ten readings before cutoff \\
\hline TwoMostRecentConcentrationSD & SD of concentration for the two readings before cutoff \\
\hline FiveMostRecentConcentrationSD & SD of concentration for the five readings before cutoff \\
\hline TenMostRecentConcentrationSD & SD of concentration for the ten readings before cutoff \\
\hline NbPriorSGenChange & Number of S Gen changes before cutoff (since start of QC sample) \\
\hline NbPriorSLotChange & Number of S Lot changes before cutoff \\
\hline NbPriorERFLotChange & Number of ERF Lot changes before cutoff \\
\hline NbPriorIWFLotChange & Number of IWF Lot changes before cutoff \\
\hline NbPriorContLotNumChange & Number of Control Lot Number changes before cutoff \\
\hline NbPriorCalCurveChange & Number of Calibration Curve changes before cutoff \\
\hline TimeSinceLastSGenChange & Time elapsed since last S Gen change before cutoff \\
\hline TimeSinceLastSLotChange & Time elapsed since last S Lot change before cutoff \\
\hline TimeSinceLastERFLotChange & Time elapsed since last ERF Lot change before cutoff \\
\hline TimeSinceLastIWFLotChange & Time elapsed since last IWF Lot change before cutoff \\
\hline TimeSinceLastContLotNumChange & Time elapsed since last Control Lot Number change before cutoff \\
\hline TimeSinceLastCalCurveChange & Time elapsed since last Calibration Curve change before cutoff \\
\hline TimeToComplain & Time elapsed since last e-Connectivity log before cutoff \\
\hline
\end{tabular}




\section{Results}

\section{Very Low Error Rates Even With Noisy Data}

To predict which call areas are used when a customer complains only using QC data (Figure 1), we implemented two machine-learning algorithms that we ran on five different assays. As expected, CART showed error rates that were higher than those obtained with adaptive boosting, but both algorithms did much better than chance, with median error rates as small as 8\% (Multimedia Appendix 5). Over the 90-day sample, each assay had triggered different numbers of complaints (assay A: 200, assay B: 835 , assay C: 227 , assay D: 182 , assay E: 410 , Multimedia Appendix 6), so that we expected that assays with larger number of complaints would have larger predictive power, but that was not the case $\left(t_{3}=1.027, P=.38\right)$. Instead, the temporal dynamics of customer complaints, which increased in the second half of the 90-day period (Figure 2), affected error rates (Multimedia Appendix 5): in particular, the first quartile of the empirical cumulative distribution of customer complaints was a strong predictor of the error rate (adaptive boosting: $t_{3}=4.103$, $P=.03$ ). This suggests that it is easier to predict a call area (the type of a problem) for assays that quickly generate complaints.

\section{Importance of Timing and Variability in Predicting Call Type}

Adaptive boosting computes a measure of importance for each feature. Multimedia Appendix 6 shows that time to complain was the most important feature across all five assays tested. The second most important features were mostly those involving the timing of maintenance events, followed by the variability of concentrations (SDs) of the QC material. Unexpectedly, the actual concentration means (last two, five, or ten) were systematically the least important features for predicting call areas.

\section{Misclassification Increases When Time to Complain Is Ignored}

The previous results included time to complain as a feature; again, this is the time lag between the last QC reading by the system and the time when a customer placed a complaint (Figure $1)$. This is unrealistic, as in a real application, we would not know when a customer is going to complain. As a result, we assessed the impact of removing the time to complain feature from our classifiers. Both CART and adaptive boosting were affected by this removal, even if all five assays still had median error rates $<50 \%$ and as low as $20 \%$ with adaptive boosting (Multimedia Appendix 5). This increase in error rate after removal of this feature shows that time to complain is an important determinant of a complaint, which in turn suggests that customers are quick to complain after detecting a QC shift.

Note, however, that this removal of the most important feature did not affect the relative importance of the other features: those involved in the timing of maintenance events and those describing the variability of concentrations (SDs) were still the most important predictors (Multimedia Appendix 7).

Figure 2. Empirical cumulative distribution function (ECDF) of customer complaints. The ECDF was plotted for the five assays considered. The horizontal gray bars represent the first, second, and third quartiles. Each assay is color-coded as shown (inset).

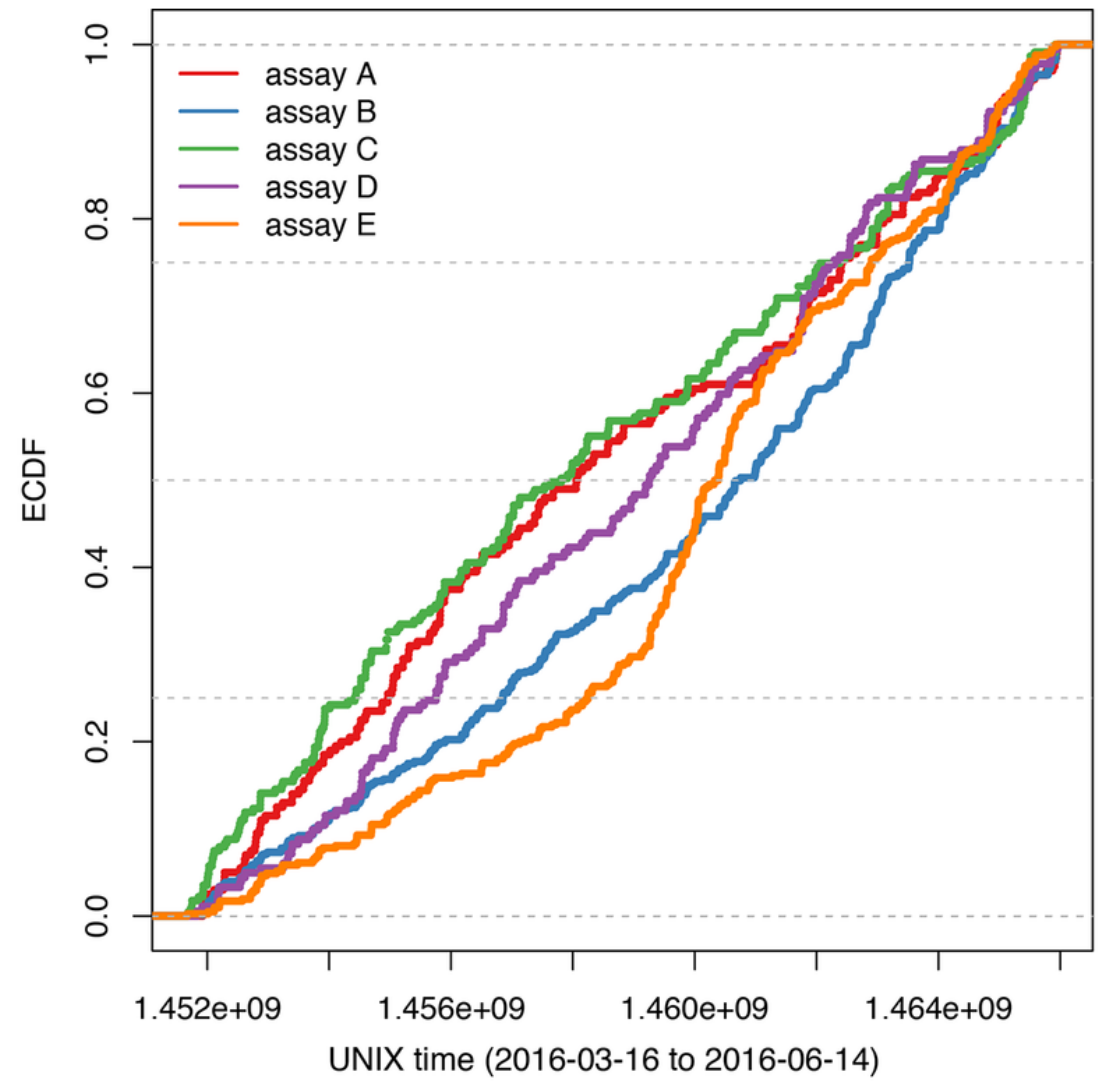




\section{Shorter Datasets Increase Accuracy}

The results above suggest that the rate of complaint may affect performance. But it is unclear if longer training periods can benefit the performance of our algorithms. To test this, we subset the 90-day data to its last 45 days. When all the features were used to train the algorithms, all classification error rates decreased (Multimedia Appendix 5). A consistent pattern was observed when time to complain was also removed from the feature list (Multimedia Appendix 5). This suggests that the statistical process underlying call areas is nonstationary in time (ie, is time-heterogeneous). This hypothesis was supported by the change in error rate of assay E, which was the worst performer with the 90-day data but became one of the best one with the 45-day data, where a sharp increase in customer complaints can be observed at the beginning of this period (Figure 2, and Multimedia Appendix 6). It is therefore possible that training periods might have to be adjusted as calls are coming in: small number of complaints may require longer training periods, whereas an increase in complaint volume may necessitate reducing the training period in real time. On the other hand, it is also clear from Multimedia Appendix 6 that by shortening the training period, the number of call types was also reduced, so that the algorithms needed to predict fewer categories, which also contributed to lowering error rates. Therefore, shorter datasets may increase accuracy, but at the cost of being less general in the type of calls that can be predicted.

\section{Data Denoising Increases Accuracy}

In an attempt to denoise the customer data, we first removed non-QC related complaints and trained our classifiers on both the 90 - and the 45-day datasets. This led to decreased error rates over all five assays (Multimedia Appendix 8), with some assays benefiting better than others (see assay D vs B) and to similar most important features (Multimedia Appendix 9). Note that for assay $\mathrm{C}$, the small volume of complaints as observed in Figure 3 led to difficulties in training both classifiers on the 45-day data, and results for this assay at this shorter time frame are therefore absent. A closer examination of the confusions tables in this case suggests that no pattern exists in how errors are generated: some assays such as B can fail to predict almost $16 \%$ of accuracy high (indicating that the measured concentration is suspected of being higher than the actual value) call areas, whereas others such as E may have a bias in overpredicting QC high (Multimedia Appendix 10). Similarly, binning the QC-high or QC-low data on the QC-only complaints led to further improvements, leading in some cases to classifiers with a zero error rate (eg, see assay A in Figure 3).

In this case, where data are denoised by binning and by only considering QC-only data, the most important features for the classifier based on adaptive boosting remain TimeToComplain for both the 90- and the 45-day datasets (Figure 4). When this feature is removed, timing of events and variability of QC logs remain the most critical factors in determining call areas of customer complaints.

Figure 3. Distribution of prediction error rates for the binned quality check (QC)-only data. Error rates are shown as derived from the cross-validation analyses, where the data were split 2500 times (see Methods). Results are shown for both classifiers, Classification and Regression Trees (CART; broken lines) and adaptive boosting (solid lines), over the five assays considered for the 90-day data with all features (a) or with TimeToComplain removed (b) and likewise for the 45-day data with (c) or not (d) all features. Each assay is color-coded as shown.

(a)
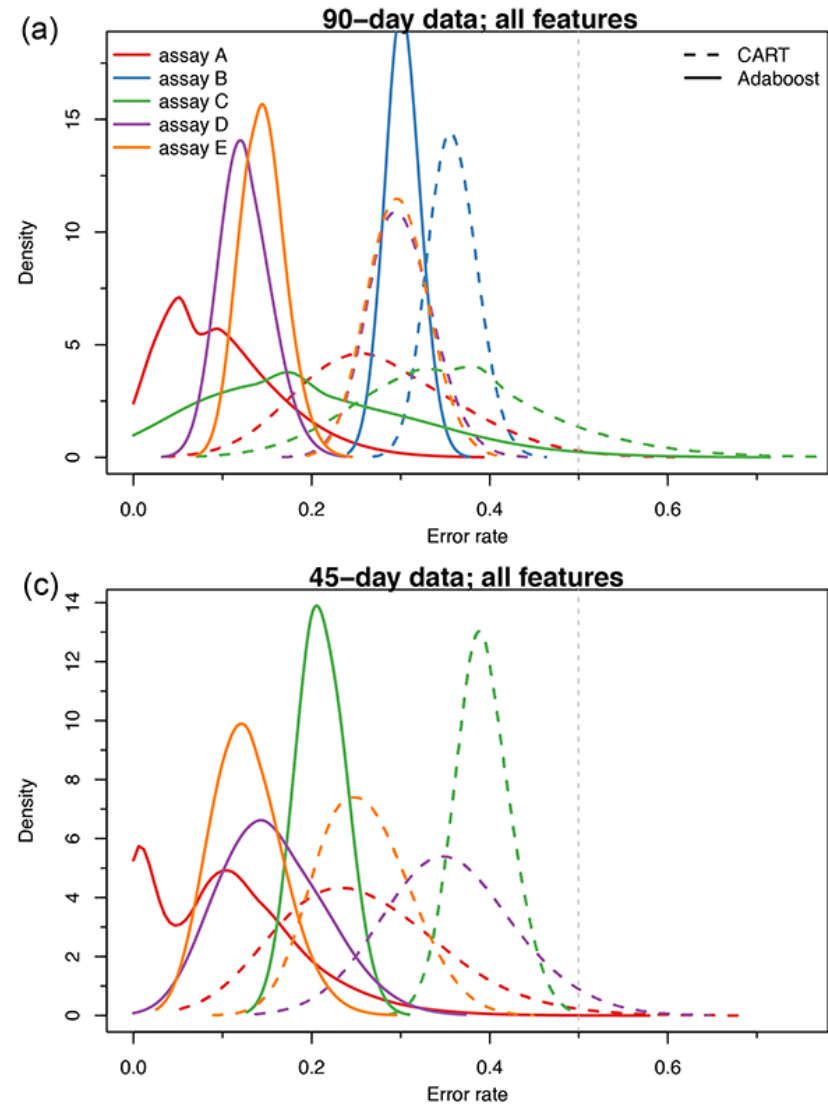

(b)

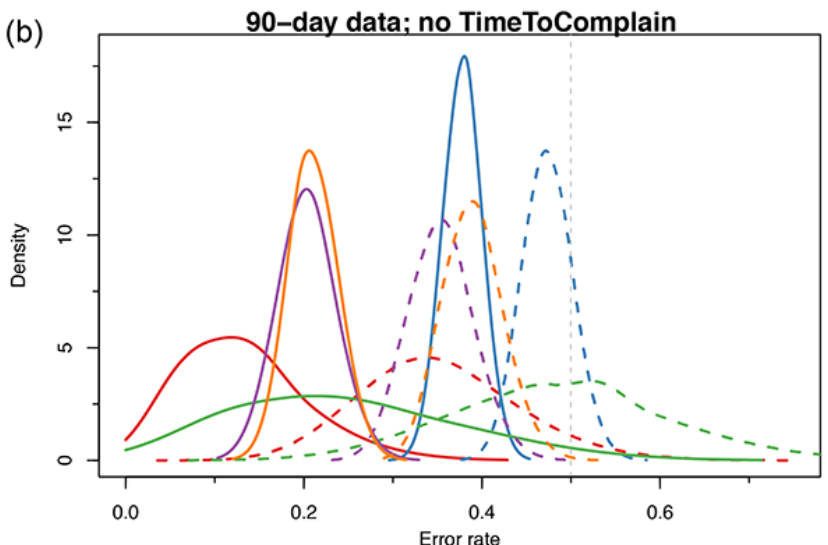

(d)

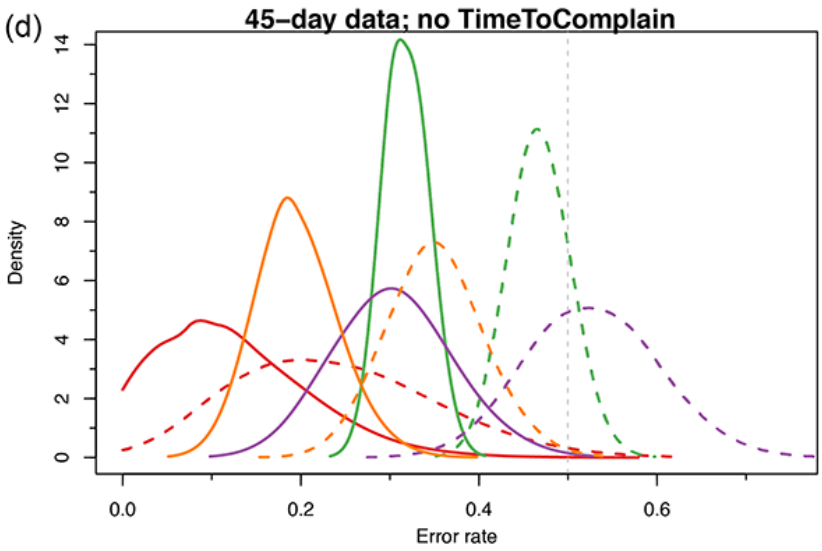


Figure 4. Feature importance under adaptive boosting for the binned quality control (QC)-only data. Importance of the features are shown as radar charts, over the five assays considered. Each assay is color-coded as shown. Top panels are for the whole 90-day datasets, whereas the bottom panels are for the 45-day datasets. Left panels include all feature; right panels exclude TimeToComplain from the models.

(a)

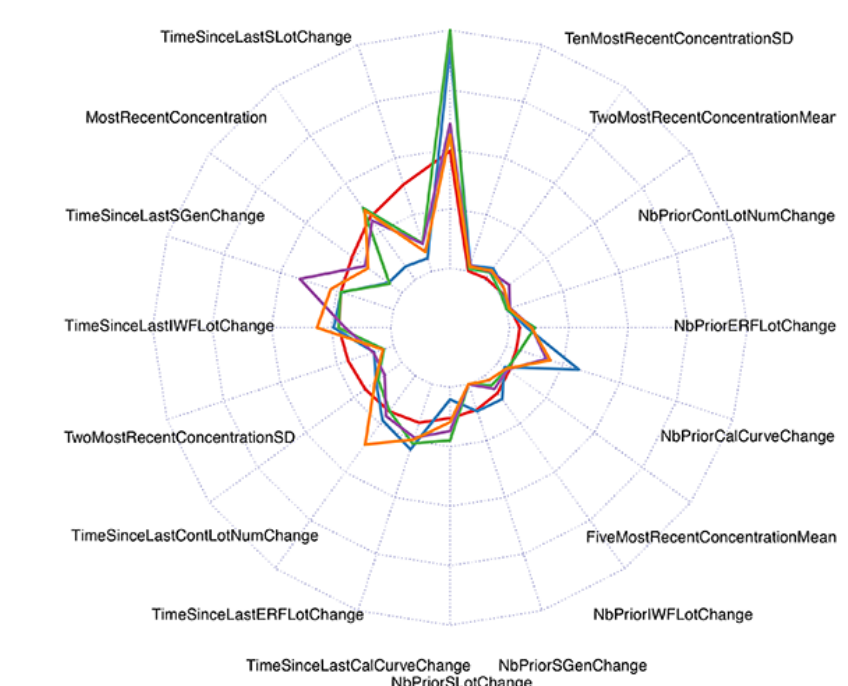

(c)

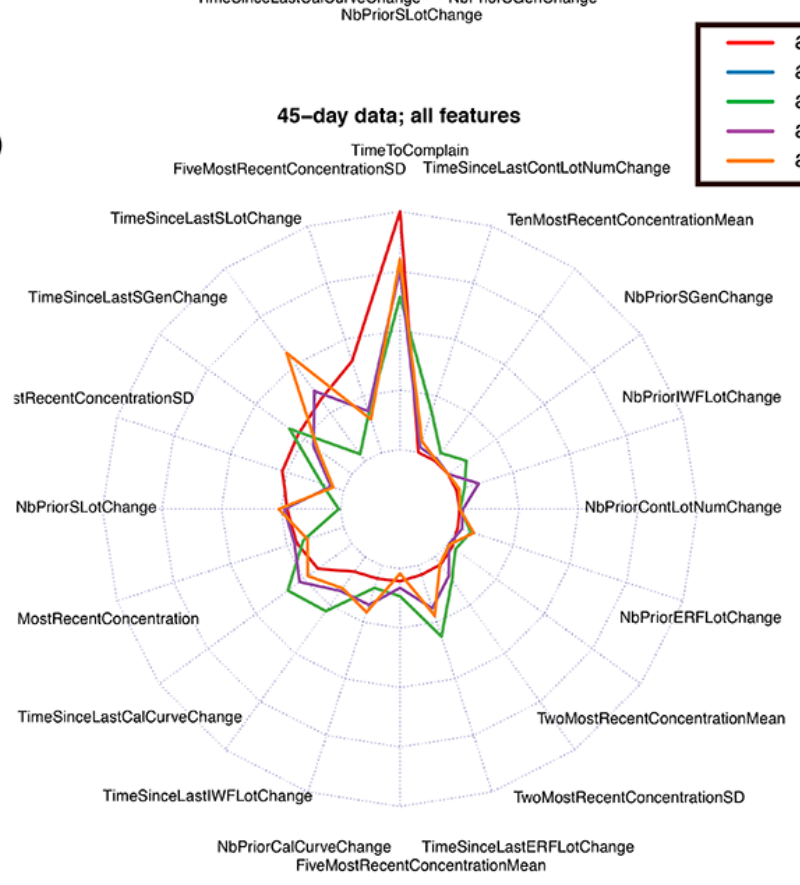

\section{Discussion}

\section{Principal Findings}

Traditionally, failure prediction in industrial applications aims at predicting when a particular system is likely to fail $[29,30]$. Here, we addressed a different question, one not directly related to the timing of failure, but one that focused on which type of failure can be predicted based on customer complaints (Figure 1). This is critical in the health care industry as it can point as to where along the process (from assay production to delivery, to storage, to use, to service on the analyzers) a product issue occurred, and hence, to take remedial steps to avoid further costs-and customer complaints. For this prediction of call areas, we compared two machine-learning algorithms, one based on decision trees (CART) and a more sophisticated one, adaptive boosting, that combines weak classifiers to produce a strong

$$
\text { 90-day data; no TimeToComplain }
$$$$
\begin{aligned}
& \text { FiveMostRecentConcentrationSD } \\
& \text { TimeSinceLastSGenChange TenMostRecentConcentrationMean }
\end{aligned}
$$
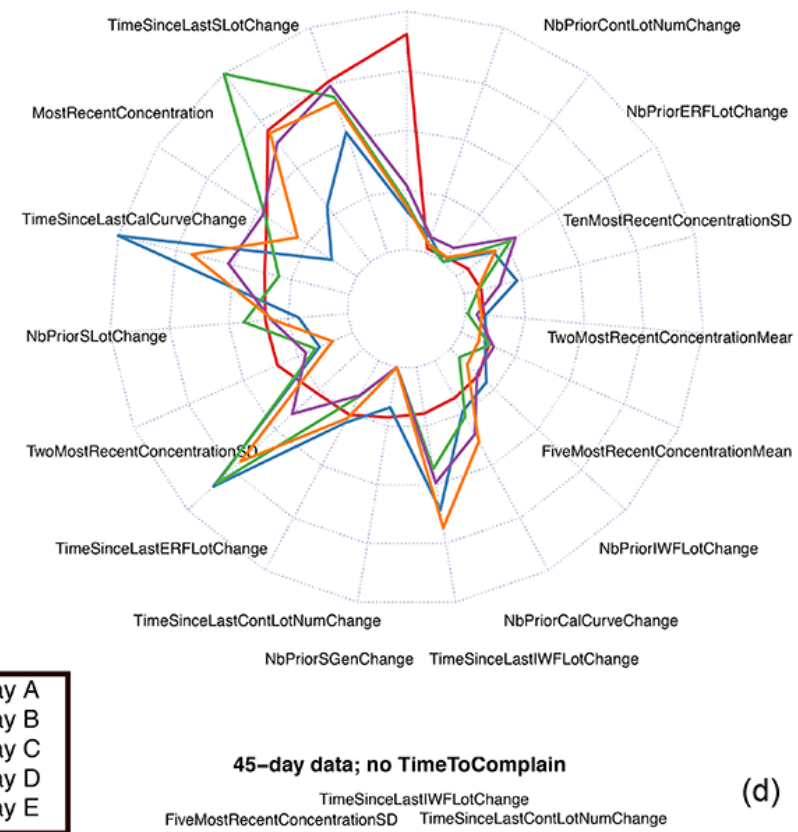

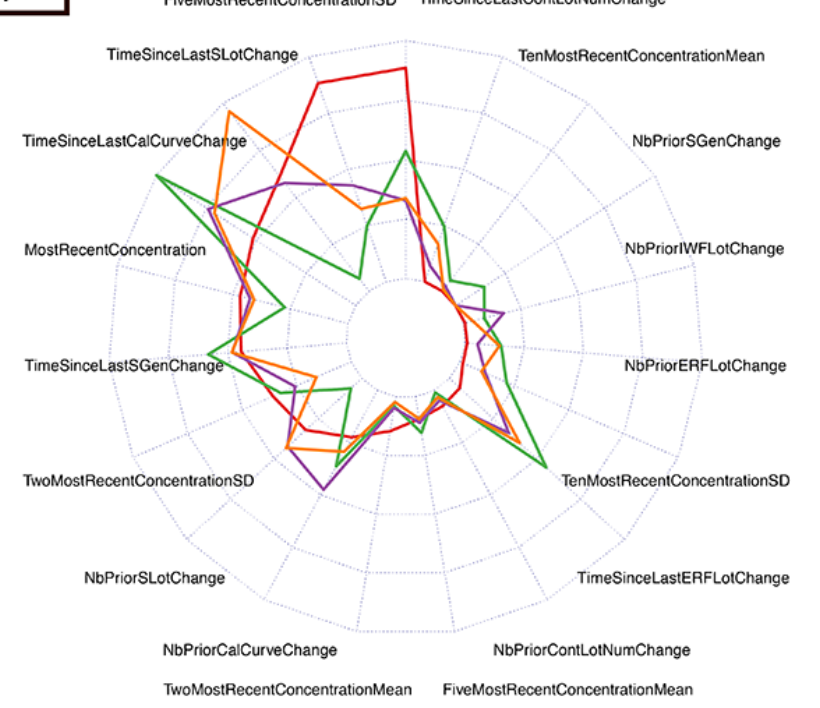

one. We showed that median errors rates can be as low as $8 \%$-while still being as low as $20 \%$ in more realistic settings, where it is unknown when a customer is going to complain-and very close to $0 \%$ after denoising of the customer data (Figure 2 ). Note that not knowing when a customer complains does not seem to affect performance order on the five assays tested here.

One of our challenges here is that a complaint is a symptom of an actual product issue. When an issue occurs, the customer may complain, or not. The customer may wait to have several incidences of same issue before complaining, or may choose not to complain because he or she is busy or stopped complaining when it is a recurrent problem. It is also possible that a customer complains when there is no product-related issue. As a result, the complaint database that we used is intrinsically noisy, but (1) This database represents the best data available and (2) The manufacturer's goal is to improve 
customer satisfaction by being able to identify issues before (or even without) a complaint call is placed.

To achieve this goal, we resorted to machine learning. As in any machine-learning application - except maybe with some deep-learning applications as those trained directly on images at the pixel level [31] — a key element is the identification and definition of the features used to train a classifier [32,33]. Rather than selecting features in an $a d-h o c$ manner, as is often the case with EHRs [34], we took inspiration from standard recordings logged by a connected system to identify features that can easily be extracted from the data and that are also likely to reflect QC shifts (Figure 1). This led us to identify two kinds of features: those based on concentration readings and those based on the timing of maintenance events. In the context of this particular manufacturer in the health care industry (Ortho Clinical Diagnostics), we showed that timing of events represent the most important features in predicting a call type in a realistic setting (were the time when a customer complains is unknown), followed by variability in concentration readings (Figure 4). A future improvement of our approach could attempt to perform unsupervised feature learning, as done in deep learning [17]. This might circumvent the following difficulties: different data types (eg, patient health instead of QC data), equipment (eg, Bio-Rad [Hercules, California] rather than Ortho Clinical Diagnostics), or logging system (eg, Bio-Rad's UnityConnect vs e-Connectivity), which might require the definition of alternative features. However, it is likely that (1) All connectivity solutions log similar chemistry end points (concentrations, timing of service, etc) and that (2) Sophisticated machine-learning algorithms such as adaptive boosting will still produce quite impressive results. Here, we did not evaluate other algorithms such as support vector machines [14], neural networks [13] or deep learning [17], or others, as most of these approaches have the same goals and can behave equally well [35].

\section{Limitations}

Some additional questions and limitations remain, however. First, we extracted data for a period of 90 days and showed that the length of this period could affect performances. Indeed, shorter training periods seem to improve prediction performances when complaint rate is high. If complaint volume does affect performance, the length of the period used for analytics should be optimized in real time. This point was not addressed here and will require further investigation, in particular, to better understand the link between the volume of customer complaints for specific call areas, the features that become the most important, and how prediction performances are affected (Figure 4). Second, we only focused on five assays and showed that our general approach seems to deliver similar performances across those particular assays. However, this need not be the case, and a systematic survey should be undertaken. Third, we employed only one particular system here, the VITROS System, manufactured by Ortho Clinical Diagnostics. However, it is not immediately clear whether our approach can be ported to other systems, be they distributed by the same or by other manufacturers. Yet, it may be expected that most systems from most manufacturers will log QC data in a similar way, which can be interpreted in the same way as here (see feature definitions). Fourth, we were limited in our analysis of QC data by not having access to actual verifiers from the manufacturer. This forced us to resort to changes in the measured concentrations, rather than simply checking departures between measurements and verifiers. However, obtaining these data was in our case challenging, as these data were only available as individual PDF files for each performance verifier lot (there were hundreds of lots). Obtaining information about these verifiers would help train our predictive tools. Fifth, we exclusively focused on QC data used for monitoring health care systems, not on patients' health. This was done to avoid complications linked to obtaining consent forms from patients in hospitals that are themselves scattered around the world. Eventually, health care analytics should also monitor individual patients and hence, help physicians in their diagnosis. Sixth, call areas, which we aimed at predicting, are used by a manufacturer to identify an issue with the product or with the analyzer in the complaint handling process: they are not the root cause of the issue, which can only be determined through what is known as a root cause investigation (RCI). RCIs are, however, very time consuming to conduct, especially on analyzers that are distributed globally, so that most of the time, the actual cause of a reported issue may not be known. However, knowing which issue may arise (ie, our prediction of call areas) instead of the actual cause can help manufacturers to initiate targeted RCIs more proactively. Finally, we have only presented one side of the health care analytics in predicting call areas, not when failures occur. An integrated solution should put together both questions, possibly by merging our approach with traditional time series methods [29,30].

In the future, a more agnostic approach with respect to feature definition may be required: indeed, the features that are based on concentration readings all depend, to some extent, on the exact time when a customer complained. This time is unknown when performing real-time analytics. To circumvent this limitation, it might be better to implement a sliding window, defined over a time period $T=\left[t_{0}, t_{1}\right]$, and use time $t=t_{1}$ as the cutoff point to define features that are based on the timing of events.

\section{Conclusions}

Although the approach we described will require further validation and testing, the ultimate goal is to implement this kind of predictive tool into the global monitoring system of IVD analyzers to help manufacturers be more proactive in detecting quality issues of the various assays they marketed around the world. This may help them pinpoint where in the manufacturing process issues are likely to originate-eg, if only a particular lot number is globally generating the same call area, a manufacturing problem specific to this lot can be identified. As such, we might one day be able to develop automated analytics or systems that can not only identify when and how failures will occur but also automatically take remediation steps to resolve these issues, in real time, without the intervention of any human being [5].

In the meantime, the US FDA is planning to use big data to guide regulatory decisions [24]. Consequently, medical companies will soon have to harness all the data logged by their 
instruments and use these data to their full potential to further improve the health care system. Our contribution here is a first step in this direction, laying ground to predicting call areas, and hence, enabling manufacturers, the expected end user of our approach, to be more proactive in postmarket surveillance. We predict that by combining our machine-learning approach with traditional time series analysis, we will eventually be able to predict when a customer will complain, in addition to what he or she will complain about. This work paves the way to developing an automated tool to anticipating customer complaints and identifying product quality issues through connected systems.

\section{Acknowledgments}

The authors would like to thank the Center for Advanced Computing and Compute Ontario for providing them with computing time; Jennifer Paine, Ian Wells, and the Safety Risk Management and Surveillance (SRMS) team for their support; Tom Balland and Craig Ritson for their help accessing the data; and Greg Munro, Jeanette Owejan, and Mike Torpey for discussions, as well as two anonymous reviewers for providing them with constructive comments. This work was supported by the Natural Sciences Research Council of Canada (SAB) and was part of Ortho Clinical Diagnostics SRMS project \#2016-0825 (SAB, JK, LL, HL). This work was completed while SAB was being hosted by Yutaka Watanuki, at the University of Hokkaido in Hakodate, thanks to an Invitational Fellowship from the Japanese Society for the Promotion of Science.

\section{Conflicts of Interest}

None declared.

\section{Multimedia Appendix 1}

Detailed description of the e-Connectivity features.

[PDF File (Adobe PDF File), 48KB-Multimedia Appendix 1]

\section{Multimedia Appendix 2}

Detailed description of the customer features.

[PDF File (Adobe PDF File), 38KB-Multimedia Appendix 2]

\section{Multimedia Appendix 3}

Description of the 99 error codes reported by the analyzers over the five assays.

[PDF File (Adobe PDF File), 127KB-Multimedia Appendix 3]

\section{Multimedia Appendix 4}

Distribution of mean concentration reading per sample for the same assay. For each sample in the e-Connectivity data, the mean of all concentration readings was taken, and their distribution over the entire e-Connectivity 90-day data set was plotted. This distribution is multimodal; modes were estimated and are shown as vertical red dotted lines.

[PDF File (Adobe PDF File), 9KB-Multimedia Appendix 4]

\section{Multimedia Appendix 5}

Distribution of prediction error rates for the unfiltered customer data. Error rates are shown as derived from the cross-validation analyses, where the data were split 2500 times (see Methods). Results are shown for both classifiers, CART (broken lines) and adaptive boosting (solid lines), over the five assays considered, for the 90-day data with all features (a) or with TimeToComplain removed (b), and likewise for the 45-day data with (c) or not (d) all features. Each assay is color-coded as shown.

\section{[PDF File (Adobe PDF File), 237KB-Multimedia Appendix 5]}

\section{Multimedia Appendix 6}

Distribution of call areas for each assay. Distributions are shown for the whole 90-day data sets (a) and the 45-day data set (b). Each assay is color-coded as shown. Non-QC related call areas were filtered out.

[PDF File (Adobe PDF File), 163KB-Multimedia Appendix 6] 


\section{Multimedia Appendix 7}

Feature importance under adaptive boosting for the unfiltered customer data. Importance of the features are shown as radar charts, over the five assays considered. Each assay is color-coded as shown. Top panels are for the whole 90-day data sets, while the bottom panels are for the 45-day data sets. Left panels include all feature, right panels exclude TimeToComplain from the models.

[PDF File (Adobe PDF File), 254KB-Multimedia Appendix 7]

\section{Multimedia Appendix 8}

Distribution of prediction error rates for the QC-only customer data. Error rates are shown as derived from the cross-validation analyses, where the data were split 2500 times (see Methods). Results are shown for both classifiers, CART (broken lines) and adaptive boosting (solid lines), over the five assays considered, for the 90-day data with all features (a) or with TimeToComplain removed (b), and likewise for the 45-day data with (c) or not (d) all features. Each assay is color-coded as shown.

[PDF File (Adobe PDF File), 233KB-Multimedia Appendix 8]

\section{Multimedia Appendix 9}

Feature importance under adaptive boosting for the QC-only customer data. Importance of the features are shown as radar charts, over the five assays considered. Each assay is color-coded as shown. Top panels are for the whole 90-day data sets, while the bottom panels are for the 45-day data sets. Left panels include all feature, right panels exclude TimeToComplain from the models.

[PDF File (Adobe PDF File), 248KB-Multimedia Appendix 9]

\section{Multimedia Appendix 10}

Examples of confusion tables obtained during cross-validation on the 90-day data, filtered for quality control (QC)-only call areas (data not binned by QC level). Numbers on the diagonal show accurate predictions; false predictions are below the diagonal, whereas missed predictions are above.

[PDF File (Adobe PDF File), 65KB-Multimedia Appendix 10]

\section{References}

1. Porter M, Heppelmann J. Harvard Business Review. 2014. How smart, connected products are transforming competition URL: https://hbr.org/2015/10/how-smart-connected-products-are-transforming-companies.pdf[WebCite Cache ID 6z8So28JG]

2. Raghupathi W, Raghupathi V. An overview of health analytics. J Health Med Informat 2013;04(03):2. [doi: 10.4172/2157-7420.1000132]

3. Alexandru A, Alexandru C, Coardos D, Tudora E. Healthcare, big data and cloud computing. URL: https://pdfs. semanticscholar.org/cdc8/deed19d2269db56e86d77f74ff7bd54b10c8.pdf[WebCite Cache ID 6z8SzRsWr]

4. R-project. 2015. R: A Language and Environment for Statistical Computing URL: https://www.R-project.org/ [accessed 2018-04-19] [WebCite Cache ID 6ymbONZ08]

5. Davenport TH. The Wall Street Journal. 2015. The rise of automated analytics URL: http://www.tomdavenport.com/ wp-content/uploads/The-Rise-of-Automated-Analytics.pdf[WebCite Cache ID 6z8T4ZuW2]

6. Vasikaran S. Anatomy and history of an external quality assessment program for interpretative comments in clinical biochemistry. Clin Biochem 2015 May;48(7-8):467-471. [doi: 10.1016/j.clinbiochem.2014.12.014] [Medline: 25543064]

7. Wilson A, Roberts W, Pavlov I, Fontenot J, Jackson B. Patient result median monitoring for clinical laboratory quality control. Clin Chim Acta 2011 Jul 15;412(15-16):1441-1446. [doi: 10.1016/j.cca.2011.04.024] [Medline: 21549689]

8. Van Houcke S, Stepman H, Thienpont L, Fiers T, Stove V, Couck P, et al. Long-term stability of laboratory tests and practical implications for quality management. Clin Chem Lab Med 2013 Jun;51(6):1227-1231. [doi:

10.1515/cclm-2012-0820] [Medline: 23337057]

9. Fleming J, Katayev A. Changing the paradigm of laboratory quality control through implementation of real-time test results monitoring: for patients by patients. Clin Biochem 2015 May;48(7-8):508-513. [doi: 10.1016/j.clinbiochem.2014.12.016] [Medline: 25549976]

10. Goossens K, Van Uytfanghe K, Twomey PJ, Thienpont LM, Participating Laboratories. Monitoring laboratory data across manufacturers and laboratories--a prerequisite to make "Big Data" work. Clin Chim Acta 2015 May 20;445:12-18. [doi: 10.1016/j.cca.2015.03.003] [Medline: 25771106]

11. Jørgensen LM, Hansen S, Petersen P, Sölétormos G. Median of patient results as a tool for assessment of analytical stability. Clin Chim Acta 2015 Jun 15;446:186-191. [doi: 10.1016/j.cca.2015.04.024] [Medline: 25920692]

12. Ferreira CE, França CN, Correr C, Zucker M, Andriolo A, Scartezini M. Clinical correlation between a point-of-care testing system and laboratory automation for lipid profile. Clin Chim Acta 2015 Jun 15;446:263-266. [doi: 10.1016/j.cca.2015.04.036] [Medline: 25952166] 
13. Ghavami P. An Investigation of Applications of Artificial Neural Networks in Medical Prognostics. Washington: University of Washington; 2012.

14. Lidbury B, Richardson A, Badrick T. Assessment of machine-learning techniques on large pathology data sets to address assay redundancy in routine liver function test profiles. Diagnosis (Berl) 2015 Feb 01;2(1):41-51. [doi: 10.1515/dx-2014-0063] [Medline: 29540013]

15. Judson R, Elloumi F, Setzer R, Li Z, Shah I. A comparison of machine learning algorithms for chemical toxicity classification using a simulated multi-scale data model. BMC Bioinformatics 2008 May 19;9(1):241 [FREE Full text] [doi: 10.1186/1471-2105-9-241] [Medline: 18489778 ]

16. Freund Y, Schapire R. Experiments with a new boosting algorithm. In: Machine Learning: Proceedings of the Thirteenth International Conference. San Francisco, CA: ICML; 1996:148-156.

17. Miotto R, Li L, Kidd B, Dudley J. Deep patient: an unsupervised representation to predict the future of patients from the electronic health records. Sci Rep 2016 Dec 17;6:26094 [FREE Full text] [doi: 10.1038/srep26094] [Medline: 27185194]

18. DiBardino D, McElhinney D, Kaza A, Mayer J. Analysis of the US Food and Drug Administration Manufacturer and User Facility Device Experience database for adverse events involving Amplatzer septal occluder devices and comparison with the Society of Thoracic Surgery congenital cardiac surgery database. J Thorac Cardiovasc Surg 2009 Jun;137(6):1334-1341 [FREE Full text] [doi: 10.1016/j.jtcvs.2009.02.032] [Medline: 19464444]

19. Noble D, Pronovost P. Underreporting of patient safety incidents reduces health care's ability to quantify and accurately measure harm reduction. J Patient Saf 2010 Dec;6(4):247-250. [Medline: 21500613]

20. Varallo F, Guimarães Synara de Oliveira P, Abjaude S, Mastroianni P. [Causes for the underreporting of adverse drug events by health professionals: a systematic review]. Rev Esc Enferm USP 2014 Aug;48(4):739-747 [FREE Full text] [Medline: 25338257]

21. de Boissieu P, Kanagaratnam L, Abou TM, Roux M, Dramé M, Trenque T. Notoriety bias in a database of spontaneous reports: the example of osteonecrosis of the jaw under bisphosphonate therapy in the French national pharmacovigilance database. Pharmacoepidemiol Drug Saf 2014 Sep;23(9):989-992. [doi: 10.1002/pds.3622] [Medline: 24737486]

22. Motola D, Vargiu A, Leone R, Conforti A, Moretti U, Vaccheri A, et al. Influence of regulatory measures on the rate of spontaneous adverse drug reaction reporting in Italy. Drug Saf 2008;31(7):609-616. [Medline: 18558794]

23. Reader TW, Gillespie A, Roberts J. Patient complaints in healthcare systems: a systematic review and coding taxonomy. BMJ Qual Saf 2014;23(8):678-689. [doi: 10.1136/bmjqs-2013-002437]

24. Food and Drug Administration. 2017. Regulatory science priorities (FY 2017) URL: https://www.fda.gov/downloads/ MedicalDevices/ScienceandResearch/UCM521503.pdf[WebCite Cache ID 6ymdBGozJ]

25. James G, Witten D, Hastie T, Tibshirani R. An introduction to statistical learning. New York: Springer; $2013: 6$.

26. Shoji A, Aris-Brosou S, Culina A, Fayet A, Kirk H, Padget O, et al. Breeding phenology and winter activity predict subsequent breeding success in a trans-global migratory seabird. Biol Lett 2015 Oct;11(10) [FREE Full text] [doi: 10.1098/rsbl.2015.0671] [Medline: 26510674]

27. Ripley B. CRAN.R-project. 2016. tree: Classification and Regression Trees URL: http://cran.r-project.org/web/packages/ tree/index.html [accessed 2018-05-03] [WebCite Cache ID 6z8Tx8g10]

28. Alfaro E, Gámez M, García N. adabag: an R package for classification with boosting and bagging. J Stat Soft 2013;54(2):35. [doi: $10.18637 /$ jss.v054.i02]

29. García F, Pedregal D, Roberts C. Time series methods applied to failure prediction and detection. Reliab Eng Syst Safe 2010 Jun;95(6):698-703. [doi: 10.1016/j.ress.2009.10.009]

30. Moura MD, Zio E, Lins I, Droguett E. Failure and reliability prediction by support vector machines regression of time series data. Reliab Eng Syst Safe 2011 Nov;96(11):1527-1534. [doi: 10.1016/j.ress.2011.06.006]

31. Esteva A, Kuprel B, Novoa R, Ko J, Swetter S, Blau H, et al. Dermatologist-level classification of skin cancer with deep neural networks. Nature 2017 Jan 25;542(7639):115-118. [doi: 10.1038/nature21056] [Medline: 28117445]

32. Bengio Y, Courville A, Vincent P. Representation learning: a review and new perspectives. IEEE Trans Pattern Anal Mach Intell 2013 Aug;35(8):1798-1828. [doi: 10.1109/TPAMI.2013.50] [Medline: 23787338]

33. Jordan M, Mitchell T. Machine learning: trends, perspectives, and prospects. Science 2015 Jul 17;349(6245):255-260. [doi: 10.1126/science.aaa8415] [Medline: 26185243]

34. Jensen P, Jensen L, Brunak S. Mining electronic health records: towards better research applications and clinical care. Nat Rev Genet 2012 May 02;13(6):395-405. [doi: 10.1038/nrg3208] [Medline: 22549152]

35. Bellazzi R, Zupan B. Predictive data mining in clinical medicine: current issues and guidelines. Int J Med Inform 2008 Feb;77(2):81-97. [doi: 10.1016/j.ijmedinf.2006.11.006]

\section{Abbreviations}

CART: Classification and Regression Trees

EHR: electronic health record

FDA: Food and Drug Administration

IVD: in vitro diagnostic 
QC: quality control

RCI: root cause investigation

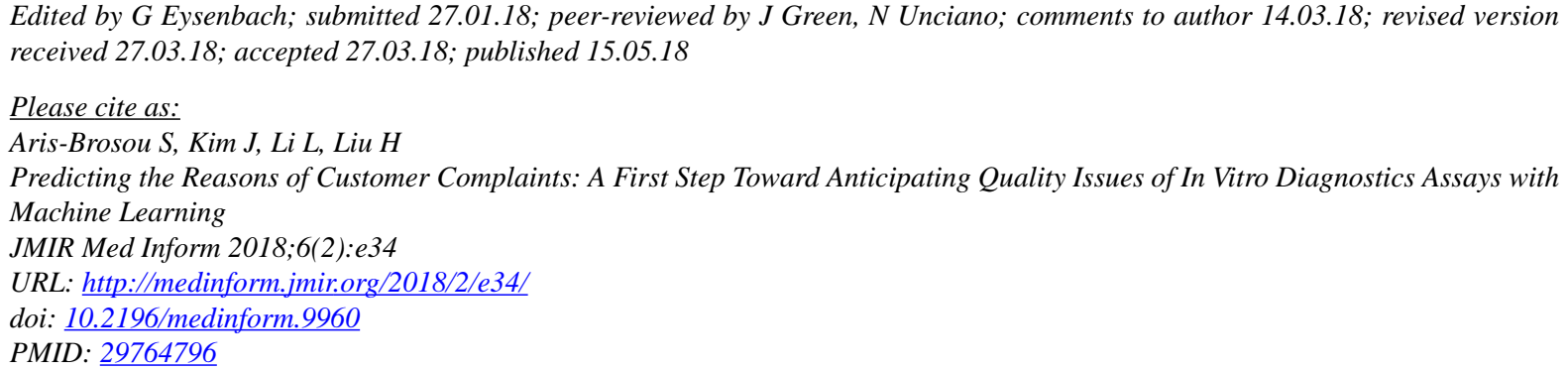

(C)Stephane Aris-Brosou, James Kim, Li Li, Hui Liu. Originally published in JMIR Medical Informatics (http://medinform.jmir.org), 15.05.2018. This is an open-access article distributed under the terms of the Creative Commons Attribution License (https://creativecommons.org/licenses/by/4.0/), which permits unrestricted use, distribution, and reproduction in any medium, provided the original work, first published in JMIR Medical Informatics, is properly cited. The complete bibliographic information, a link to the original publication on http://medinform.jmir.org/, as well as this copyright and license information must be included. 